\title{
Consensus and the Act of Voting
}

\author{
J. C. R. Alcantud ${ }^{\mathrm{a}}$, R. de Andrés Calle ${ }^{\mathrm{a}}$, J. M. Cascón ${ }^{\mathrm{a}}$ \\ ${ }^{a}$ Facultad de Economía y Empresa, Universidad de Salamanca, 37007 Salamanca, Spain
}

\begin{abstract}
In this paper we are concerned with assessing the cohesiveness of a society whose individual preferences are known. We analyse the axiomatic properties of a general proposal to measure aggregate satisfaction, that relies on the consensus with reference to a select social preference.
\end{abstract}

Keywords: Consensus, measurement, Borda rule, Copeland rule

JEL classification: D39, D70.

\section{Introduction}

Consider the case of a finite society that must express its preferences on a finite list of alternatives (or more generally, that aggregates its members' preferences into a social preference on the alternatives). In this paper we are concerned with the aggregate welfare that the profile of preferences brings about to this society. We analyse the axiomatic properties of a proposal to measure such satisfaction that intends to capture the consensus that a social preference by a representative agent furnishes. Under this position the informational basis of the model (all personal preferences on the alternatives) must bear comparison with the informational structure of the social representative. Two preliminary remarks on our model are in order. For one thing, our procedure to measure the consensus (relative to the social benchmark) is in line with previous analyses like e.g., Bosch (2005) or Alcalde and Vorsatz (2010) where an absolute coefficient is sought. For another, and concerning what an "ideal" focal agent could be, much has been written about the (un)suitability of aggregation rules thus any possible choice will raise criticisms. Therefore in order to set forth the virtues of our model we expound on the normative properties of a general class of measures where the idea of a fictitious representative agent is captured by select voting rules.

The literature has provided several studies of metrics producing distance measures for pairs of individual preferences expressed by binary relations (cf., e.g., Kemeny, 1959; Kendall, 1962; Cook and Seiford, 1978, 1982; Klamler, 2008; Baldiga and Green, 2011). Concerning groups of individuals we can address to the aforementioned Bosch (2005) and Alcalde and Vorsatz (2010), and our objective resembles theirs in that we consider finite groups of agents too. But we 
must emphasize from the outset that we separate from their positions in a critical point: in the frequent situations where individual preferences are formed in order to make a collective judgement, a measure of the cohesiveness of preferences as a social welfare function must relate to the output that they might generate. To see this, suppose for example the case where pairwise collective comparisons are made on a unanimity basis (i.e., in order for " $\mathrm{x}$ " to be socially better than " $y$ " all agents must agree that " $x$ " is better than " $y$ "). Then in a large group voting on two alternatives " $x$ " and " $y$ ", if all members except one agree that " $x$ " is better than " $y$ " but one member thinks the opposite it is intuitively appealing that an absolute measure of the consensus à-la-Bosch should yield a high value although the social welfare that the preferences actually warrant seems negligible. So under a welfarist point of view we cannot detach the possibility of a collective outcome from the configuration of the preference profile. Our goal is shaped by these constraints. We present the concept of referenced consensus measures which permit to produce a numerical social evaluation from purely ordinal individual information. It can be specialized via two ways: the specification of the representative agent, and the measure of agreement between profiles of orderings and individual orderings. Then we perform a descriptive analysis of their formal properties with an emphasis on two relevant cases whose explicit constructions are detailed ${ }^{1}$. Our proposal does not intend to be univer sally applicable but we stress that introducing a fictitious agent as a reference is fit for the case of actual social choices.

The paper is organized as follows. Section 2 introduces basic notation and definitions. Furthermore in this section, our proposal of measurement of consensus, the referenced consensus measure, is introduced as well as a relevant particular subclass of referenced consensus measures, the normal referenced consensus measure. In Section 3, operational characterizations of some focal voting rules are provided, which helps us to deal with the two explicit proposals for measurement of consensus that we present. Also we perform a short analysis of the dichotomous case. In Section 4 we explore a list of appealing properties of normal referenced consensus measures, and particularly of our explicit proposals. Finally, in Section 5 we give some concluding remarks and pose questions for further research.

\section{Basic notation and definitions}

We fix $X=\left\{x_{1}, \ldots, X_{k}\right\}$, a finite set of $k$ options, alternatives or candidates. Abusing notation, on occasions we refer to option $X_{s}$ as option $S$ for convenience. A population of agents or voters is a finite subset $\mathbf{N}=\{1,2, \ldots, N\}$ of natural numbers. We also denote $\mathbf{K}=\{\{i, j\} \subseteq \mathbf{N}: i, j \in\{1,2, \ldots, k\}, i>j\}$.

\footnotetext{
${ }^{1}$ A computational comparison between two focal instances has been outlined elsewhere (Alcantud, de Andrés, Cascón, 2011).
} 
Let $W(X)$ be the set of weak orders or complete preorders on $X$, that is, the set of complete and transitive binary relations on $X$. If $R \in W(X)$ is a weak order on $X$ that reflects the preferences of a voter, then by $x_{k} R x_{j}$ we mean "R-voter thinks that alternative $x_{k}$ is at last as good as $X_{j} " L(X)$ denotes the set of linear orders on $X$.

A profile $\mathrm{R}=\left(R_{1}, \ldots, R_{N}\right) \in W(X) \times \ldots . . \times W(X)$ is a vector of weak orders, where $R_{i} \in W(X)$ represents the preferences of the individual $i$ on the $k$ alternatives or candidates for each $i=1, \ldots, N$. The reversal of the profile $\mathrm{R}$, denoted by $\mathrm{R}^{-1}$, is the profile $\left(R_{1}^{-1}, \ldots, R_{N}^{-1}\right)$ where $x_{s} R_{i}^{-1} x_{t} \Leftrightarrow x_{t} R_{i} x_{s}$ for each possible voter $i \in\{1, \ldots, N\}$ and candidates or alternatives $X_{s}, X_{t} \in\{1, \ldots, k\}$. We say that the profile $\mathrm{R}$ is constant to $R$ if $\mathrm{R}=(R, \ldots \ldots, R)$.

Any permutation $\sigma$ of the voters $\{1,2, \ldots, N\}$ determines a permutation of $\mathrm{R}$ by $\mathrm{R}^{\sigma}=\left(R_{\sigma(1)}, \ldots \ldots, R_{\sigma(N)}\right)$. Similarly, any permutation $\pi$ of the candidates $\{1,2, \ldots, k\}$ determines a permutation of every complete preorder $R \in W(X)$ via $x_{s}{ }^{\pi} R_{i} x_{t} \Leftrightarrow x_{\pi^{-1}(s)} R_{i} x_{\pi^{-1}(t)}$ for all $s, t \in\{1, \ldots, k\}$ and $i \in\{1, \ldots, N\}$. Then with $\mathrm{R}$ and $\pi$ we can associate ${ }^{\pi} \mathrm{R}=\left({ }^{\pi} R_{1}, \ldots \ldots .,{ }^{\pi} R_{N}\right)$.

Finally, given any profile of weak orders $\mathrm{R}=\left(R_{1}, \ldots, R_{N}\right) \in W(X)^{N}$ and any weak order $R^{\prime}$ on $X$, we denote $\mathrm{R} \oplus R^{\prime}$ the profile $\left(R_{1}, \ldots, R_{N}, R^{\prime}\right)$ of $N+1$ weak orders. We denote by $\mathrm{P}(X)$ the set of all profiles, that is, $\mathrm{P}(X)=\bigcup_{N \geq 1} W(X)^{N}$.

\subsection{Basic definitions}

We start by recalling some definitions from Alcantud, de Andrés, Cascón (2011).

Definition 1 A Consensus measure with reference to a consensus function (henceforth, referenced consensus measure, RCM for simplicity, when the consensus function is common knowledge $)$ is a pair $\mathbf{M}=(\mathbf{C}, \partial)$ where: 
1. C is a consensus function (McMorris and Powers, 2009), that is, a mapping

$$
\mathrm{C}: \mathrm{P}(X) \rightarrow W(X)
$$

that associates a complete preorder $C(R)$ with each profile of complete preorders $R$. We speak of the consensus preorder $\mathrm{C}(\mathrm{R})$ associated with $\mathrm{R}$, and assume that

a) $\mathrm{C}(\mathrm{R})=R$ for each profile $\mathrm{R}$ that is constant to the complete preorder $R$.

b) $\mathrm{C}\left(\mathrm{R}^{\sigma}\right)=\mathrm{C}(\mathrm{R})$ for each profile of complete preorders and $\sigma$ permutation of the voters.

c) $C\left({ }^{\pi} \mathrm{R}\right)={ }^{\pi} \mathrm{C}(\mathrm{R})$ for each profile of complete preorders and $\pi$ permutation of the candidates or alternatives.

Abusing notation, this can be replaced with a voting rule with suitable properties: for example, 1.b) and 1.c) just mean the usual anonymity and neutrality conditions, respectively.

2. $\partial$ is a referenced measure function (RMF), that is, a mapping

$$
\partial: \mathrm{P}(X) \times W(X) \rightarrow[0,1]
$$

that assigns a real number, $\partial(\mathrm{R}, R) \in[0,1]$, to each pair of a profile of complete preorder $\mathrm{R}$, and a complete preorder $R$, with the following properties:

a) $\quad \partial(\mathrm{R}, R)=1$ if and only if $\mathrm{R}$ is constant to $R$.

b) $\partial\left(\mathrm{R}^{\sigma}, R\right)=\partial(\mathrm{R}, R)$ for each possible permutation $\sigma$ of the voters.

c) $\quad \partial\left({ }^{\pi} \mathrm{R},{ }^{\pi} R\right)=\partial(\mathrm{R}, R)$ for each possible permutation $\pi$ of the candidates.

With regard to $\mathbf{M}=(\mathrm{C}, \partial)$ each profile of complete preorders $\mathrm{R}$ on $X$ has a consensus $\nabla_{\mathbf{M}}(\mathrm{R})=\partial(\mathrm{R}, \mathrm{C}(\mathrm{R}))$.

Each conventional consensus measure can be interpreted as a referenced consensus measure (Alcantud, de Andrés, Cascón, 2011, Lemma 1). For this reason it is analytically convenient to restrict ourselves to a significant proper subclass where a better normative behaviour can be 
guaranteed. To that purpose Alcantud, de Andrés and Cascón (2011) introduce the following concept:

Definition 2 A referenced consensus measure $\mathbf{M}=(\mathbf{C}, \partial)$ is called normal referenced consensus measure if its referenced measure function $\partial$ verifies

$$
\text { 2.d) } \partial(\mathrm{R}, R)>0 \text { if } R \in \mathrm{R} \text {. }
$$

If overall satisfaction is an aggregate of individual satisfaction, property $2 . d$ ) can be considered as natural.

In order to check the profiles of individual preferences against the social benchmark, the following general proposal is exploited:

Definition 3 Let $M$ be a consensus measure. Given a profile of complete preorders $\mathrm{R}$ and a complete preorder $R$ we define the $\mu^{p}(\mathrm{M})$-reference measure function $\left(\mu^{p}(\mathrm{M})-\mathrm{RMF}\right)$ as the $p$-generalized mean of the $\mathrm{R}^{N}$ vector that has the $i$-th component equal to $\mathrm{M}\left(\mathrm{R}_{i} \oplus R\right)$, that is

$$
\partial_{\mathrm{M}}^{p}(\mathrm{R}, R)=\left(\sum_{i=1}^{N} \frac{1}{N} \mathrm{M}\left(\mathrm{R}_{i} \oplus R\right)^{p}\right)^{1 / p}
$$

It is trivial to check that properties 2.a), 2.b), 2.c) and 2.d) hold true.

This parametric format can be specialized via $p$ and the consensus measure. In particular, we conclude this part with the important example of referenced measure functions given by the arithmetic mean and Kemeny's measure:

Example 1 For every profile of complete preorders $\mathrm{R}=\left(R_{1}, \ldots, R_{N}\right)$, its Kemeny's measure $\mathrm{K}(\mathrm{R})$ is the probability that the binary ordering between a pair of randomly selected alternatives is the same for all voters. Given $p=1$ and $\mathrm{M}=\mathrm{K}$, the construction above produces the following RMF. Attending to (1), we have to compute the Kemeny's measure of a profile composed by two elements: $R_{i}$ that represents the preferences of individual $i$ and $R$ the referenced complete preorder. Observe that there is a total of $\frac{k(k-1)}{2}$ possible random 
choices. Thus the proportion of pairwise comparisons where $R_{i}$ and $R$ coincide is

$$
\mathrm{K}\left(R_{i} \oplus R\right)=\frac{2}{k(k-1)} \sum_{(s, t) \in \mathbf{K}} \mathrm{K}^{s, t}\left(R_{i} \oplus R\right)
$$

with

$$
\mathrm{K}^{s, t}\left(R_{i} \oplus R\right)= \begin{cases}1 & \text { if } R_{i} \text { and } R \text { coincide on the binary comparison, } \\ & \text { between } x_{s} \text { and } x_{t}, \\ 0 \quad & \text { otherwise. }\end{cases}
$$

We then conclude that the $\mu^{1}(\mathrm{~K})$ - RMF is given by:

$$
\partial_{\mathrm{K}}^{1}(\mathrm{R}, R)=\frac{\mathrm{K}\left(R_{1} \oplus R\right)+\ldots+\mathrm{K}\left(R_{N} \oplus R\right)}{N} .
$$

Convention 1 In what follows we omit superscripts when they are 1 . We denote the $\mu^{1}(\mathrm{~K})$ RMF as $\partial_{\mathrm{K}}$.

Along the rest of the paper we restrict our attention to normal referenced consensus measure with referenced measure function based on generalized means.

\section{Some proposals for normal referenced consensus measures}

In this section we detail the construction of two relevant normal RCM proposals. These models check the profiles against the classical Borda and Copeland methods, that we proceed to recall, and they measure the consensus with the $\mu^{1}(\mathrm{~K})$ - RMF.

Example 2 A tie-breaking Borda rule (Suzumura, 1983, pp. 107-108) attaches a complete preorder to each profile of complete preorders. It ranks the candidates according to their respective Borda score defined as follows:

$$
\beta\left(x_{s}\right)=\sum_{i=1}^{N}\left(\#\left\{x_{t} \in X: x_{s} R_{i} x_{t}\right\}-\#\left\{x_{t} \in X: x_{t} R_{i} x_{s}\right\}\right) .
$$

Because 1.a), 1.b) and 1.c) are immediate, we denote by $\mathrm{C}_{\mathrm{B}}$ such consensus function.

If in fact we have a profile of linear orders the same ranking is obtained through the alternative Borda score given by:

$$
\beta^{\prime}\left(x_{s}\right)=\sum_{i=1}^{N}\left(\#\left\{x_{t} \in X: x_{s} R_{i} x_{t}\right\}\right)
$$


Example 3 The Copeland method (Saari and Merlin, 1996; Suzumura, 1983, p. 108) ranks the candidates according to their respective Copeland score defined as follows:

$$
\kappa\left(x_{s}\right)=\#\left\{x_{t} \in X: x_{s} \text { beats } x_{t} \text { by s.s.m. }\right\}-\#\left\{x_{t} \in X: x_{t} \text { beats } x_{s} \text { by s.s.m. }\right\}
$$

where s.s.m. stands for "strict simple majority". This rule is widely used in tournament situations, and versions of it are adopted by sports leagues. Again, 1.a), 1.b) and 1.c) are immediate. We denote by $\mathrm{C}_{C}$ its associated consensus function.

We proceed to provide operational characterizations of the Borda and Copeland rules. We then describe our proposals and briefly discuss the dichotomous case.

\subsection{Some operational characterizations}

Let us fix a profile $\mathrm{R}=\left(R_{1}, \ldots, R_{N}\right)$ of complete preorders on $X$. Its Borda and Copeland scores can be reinterpreted in terms of simple matrix operations. We denote by $A^{t}$ the transpose of the matrix $A$. For any $m \times n$ real-valued matrix $A=\left(a_{i, j}\right)_{m \times n}$ the notation $\operatorname{sig}(A)$ refers to the $m \times n$ matrix whose $(i, j)$ cell is 1 if $a_{i, j}>0,-1$ if $a_{i, j}<0$, and 0 otherwise. $I_{k}$ denotes the identity matrix of size $k \times k$.

For each complete preorder $R_{s}$, the asymmetric part of which is denoted by $P_{s}$, its preference matrix $\mathbf{P}_{s}$ is defined as the $k \times k$ binary matrix whose $(i, j)$ cell is 1 when $x_{i} P_{s} x_{j}$, and 0 otherwise. Observe that $R_{s}$ is linear if and only if $\mathbf{P}_{s}+\left(\mathbf{P}_{s}\right)^{t}+I_{k}=(1)_{k \times k}$, the constant to 1 matrix of size $k \times k$. The sum of the cells in the $i$-th row of $\mathbf{P}_{s}-\left(\mathbf{P}_{s}\right)^{t}$ is $\#\left\{x_{j} \in X: x_{i} P_{s} x_{j}\right\}-\#\left\{x_{j} \in X: x_{j} P_{s} x_{i}\right\}$. We say that $\mathrm{R}$ has an aggregate preference matrix $\mathbf{A}(\mathrm{R})=\mathbf{P}_{1}+\ldots+\mathbf{P}_{N}$. Its $(i, j)$ cell has the number of agents for which alternative $x_{i}$ is strictly better than $x_{j}$. The sum of the cells in its $i$-th row is the usual Borda score $\beta^{\prime}\left(x_{i}\right)$ when $\mathrm{R}$ is a profile of linear orders.

Define $\overline{\mathbf{A}}(\mathrm{R})=\mathbf{A}(\mathrm{R})-(\mathbf{A}(\mathrm{R}))^{t}$, then the sum of the cells in its $i$-th row is $\beta\left(x_{i}\right)$, the Borda score of alternative $X_{i}$ (see Example 2).

Observe that the fact that the $(i, j)$ cell of $\overline{\mathbf{A}}(\mathrm{R})$ is greater than 0 is equivalent to the fact that alternative $x_{i}$ beats $x_{j}$ by strict simple majority under the profile $\mathrm{R}$. Thus if we define 
$\widetilde{\mathbf{A}}(\mathrm{R})=\operatorname{sig}(\overline{\mathbf{A}}(\mathrm{R}))$ then the sum of the cells in its $i$-th row is $\kappa\left(x_{i}\right)$, the Copeland score of alternative $X_{i}$ (see Example 3).

Example 4 Suppose $X=\{x, y, z, w\}$ thus $k=4$. Let $\mathrm{R}=\left(R_{1}, R_{2}, R_{3}\right)$ be the profile of linear orders given by: $w P_{1} y P_{1} x P_{1} z, z P_{2} w P_{2} y P_{2} x, x P_{3} z P_{3} y P_{3} w$.

Then

$$
\mathbf{P}_{1}=\left(\begin{array}{llll}
0 & 0 & 1 & 0 \\
1 & 0 & 1 & 0 \\
0 & 0 & 0 & 0 \\
1 & 1 & 1 & 0
\end{array}\right) \quad \mathbf{P}_{2}=\left(\begin{array}{llll}
0 & 0 & 0 & 0 \\
1 & 0 & 0 & 0 \\
1 & 1 & 0 & 1 \\
1 & 1 & 0 & 0
\end{array}\right) \quad \mathbf{P}_{3}=\left(\begin{array}{llll}
0 & 1 & 1 & 1 \\
0 & 0 & 0 & 1 \\
0 & 1 & 0 & 1 \\
0 & 0 & 0 & 0
\end{array}\right)
$$

Some simple computations yield

$$
\mathbf{A}(\mathrm{R})=\left(\begin{array}{llll}
0 & 1 & 2 & 1 \\
2 & 0 & 1 & 1 \\
1 & 2 & 0 & 2 \\
2 & 2 & 1 & 0
\end{array}\right) \quad \overline{\mathbf{A}}(\mathrm{R})=\widetilde{\mathbf{A}}(\mathrm{R})=\left(\begin{array}{rrrr}
0 & -1 & 1 & -1 \\
1 & 0 & -1 & -1 \\
-1 & 1 & 0 & 1 \\
1 & 1 & -1 & 0
\end{array}\right)
$$

Thus for this setting the Borda and Copeland scores coincide throughout. Their values are -1 for options $X$ and $y$, and 1 for options $z$ and $w$. Therefore the social preference $R$ that is derived from both choice rules is $w I z P x I y$ (Suzumura, 1983, p. 108).

Calculating $\partial_{\mathrm{K}}(\mathrm{R}, R)$ for $\mathrm{R}=\left(R_{1}, \ldots, R_{N}\right)$ profile of complete preorders and $R$ complete preorder is trivial from the numbers $\mathrm{K}^{s, t}\left(R_{i} \oplus R\right)$. These amounts can be computed with the assistance of basic matrix manipulations too. Denote by $\mathbf{P}$ the preference matrix of $R$ defined as above. Let us observe two facts.

1. Cell $(s, t)$ of both $\mathbf{P}_{i}+\left(\mathbf{P}_{i}\right)^{t}$ and $\mathbf{P}+\mathbf{P}^{t}$ has a 0 if and only if both $R_{i}$ and $R$ are indifferent between $X_{s}$ and $X_{t}$. This can not happen when $s \neq t$ if either $R_{i}$ or $R$ is linear.

2. Cell $(s, t)$ of both $\mathbf{P}_{i}$ and $\mathbf{P}$ has a 1 if and only if $x_{s} P_{i} x_{t}$ and $x_{s} P x_{t}$.

This means that the number of pairs of different options for which both $R_{i}$ and $R$ are indifferent is the number of cells strictly above the diagonal with a 0 for both $\mathbf{P}_{i}+\left(\mathbf{P}_{i}\right)^{t}$ and 
$\mathbf{P}+\mathbf{P}^{t}$ (and it is 0 if either $R_{i}$ or $R$ is linear); and the number of pairs of options for which $R_{i}$ and $R$ have equal strict preference is the number of cells (outside the diagonal) with a 1 for both $\mathbf{P}_{i}$ and $\mathbf{P}^{2}$. The sum of these two amounts is $\frac{k(k-1)}{2} \mathrm{~K}\left(R_{i}, R\right)=\sum_{\{s, t\} \in \mathbf{K}} \mathrm{K}^{s, t}\left(R_{i} \oplus R\right)$.

Example 5 In the situation of Example 4 one has

$$
\mathbf{P}_{1}+\mathbf{P}_{1}^{t}=\mathbf{P}_{2}+\mathbf{P}_{2}^{t}=\mathbf{P}_{3}+\mathbf{P}_{3}^{t}=\left(\begin{array}{cccc}
0 & 1 & 1 & 1 \\
1 & 0 & 1 & 1 \\
1 & 1 & 0 & 1 \\
1 & 1 & 1 & 0
\end{array}\right)
$$

because all $\mathbf{P}_{1}, \mathbf{P}_{2}$ and $\mathbf{P}_{3}$ are linear orders. The preference matrix of the complete preorder $R$ that is prescribed by both the Borda and Copeland rule is

$$
\mathbf{P}=\left(\begin{array}{llll}
0 & 0 & 0 & 0 \\
0 & 0 & 0 & 0 \\
1 & 1 & 0 & 0 \\
1 & 1 & 0 & 0
\end{array}\right)
$$

Some simple computations yield $\partial_{\mathrm{K}}(\mathrm{R}, R)=\frac{1}{3} \cdot \frac{2+4+1}{6}=\frac{7}{18}$ since

- No pair of different options is indifferent under any of the $P_{i}$ 's.

- Only cells $(4,1)$ and $(4,2)$ have a 1 in both $\mathbf{P}_{1}$ and $\mathbf{P}$.

- Only cells $(3,1),(3,2),(4,1)$ and $(4,2)$ have a 1 in both $\mathbf{P}_{2}$ and $\mathbf{P}$.

- Only cell $(3,2)$ has a 1 in both $\mathbf{P}_{3}$ and $\mathbf{P}$.

\subsection{The RCM-B and RCM-C proposals}

The referenced consensus measure given by the tie-breaking Borda rule (cf., Example 2) and $\partial_{K}$ introduced in Example 1 is referred to as RCM-B, that is, $\mathbf{M}_{B}=\left(C_{B}, \partial_{K}\right)$. Let us now show how this proposal produces its output with a simple example.

\footnotetext{
${ }^{2}$ This number is obtained with a computer assistant very easily: do the cell-by-cell multiplication and sum up all the cells in the result.
} 
Example 6 In the situation of Example 4 we checked that $C_{B}(R)$ is the complete preorder $R_{B}$ determined by $w I_{B} z P_{B} x I_{B} y$. According to Example 5

$$
\nabla_{\mathbf{M}_{B}}(\mathrm{R})=\partial_{\mathrm{K}}\left(\mathrm{R}, \mathrm{C}_{\mathrm{B}}(\mathrm{R})\right)=\frac{7}{18}
$$

therefore 7 out of $18=\frac{3 \cdot 4 \cdot(4-1)}{2}$ possible pairwise comparisons made by a member of the society $\{1,2,3\}$ coincide with the binary ordering given by the consensus function in the model.

The referenced consensus measure given by the Copeland method (cf., Example 3 ) and $\partial_{K}$ is referred to as RCM-C, that is, $\mathbf{M}_{C}=\left(C_{C}, \partial_{K}\right)$. The following example illustrates its calculation.

Example 7 In the situation of Example 4 (see Example 6) we found $C_{C}(R)=R_{B}$ thus

$$
\nabla_{\mathbf{M}_{C}}(\mathrm{R})=\partial_{\mathrm{K}}\left(\mathrm{R}, \mathrm{C}_{\mathrm{C}}(\mathrm{R})\right)=\frac{7}{18}
$$

Again, 7 out of 18 possible pairwise comparisons made by a member of the society $\{1,2,3\}$ coincide with the binary ordering given by the consensus function in the model.

\subsection{The case of a dichotomous choice}

Suppose $k=2$, i.e., the dichotomous case. To simplify notation let $X=\{x, y\}$. We also denote $\quad n_{1}=\left|\left\{i \in \mathbf{N}: x P_{i} y\right\}\right| \quad$ and $\quad n_{2}=\left|\left\{i \in \mathbf{N}: y P_{i} x\right\}\right|, \quad$ thus $N-n_{1}-n_{2}=\left|\left\{i \in \mathbf{N}: x I_{i} y\right\}\right|$. Due to properties 1.b) and 2.b) we can reorder the voters as convenient, and we assume that voters $1, \ldots, n_{1}$ prefer $x$ strictly over $y$, that voters $n_{1}+1, \ldots, n_{1}+n_{2}$ prefer $y$ strictly over $x$, and that the last $N-n_{1}-n_{2}$ voters are indifferent between $x$ and $y$. Let $n_{x, y}(\mathrm{R})$ denote the majority margin of $x$ over $y$ under $\mathrm{R}$, that is, the number of voters that prefer $x$ strictly over $y$ minus the number of voters that prefer $y$ strictly over $x$, or $n_{x, y}(\mathrm{R})=n_{1}-n_{2}$. Now the Borda and Copeland voting rule coincide with strict simple majority: the preference matrix of the complete preorder $R_{0}$ that is prescribed by them is 


$$
\begin{aligned}
& \mathbf{P}_{\mathbf{0}}=\left(\begin{array}{ll}
0 & 1 \\
0 & 0
\end{array}\right) \text { if } n_{x, y}(\mathrm{R})>0\left(\text { or } n_{1}>n_{2}\right) \\
& \mathbf{P}_{\mathbf{0}}=\left(\begin{array}{ll}
0 & 0 \\
0 & 0 \\
&
\end{array}\right) \text { if } n_{x, y}(\mathrm{R})=0\left(\text { or } n_{1}=n_{2}\right) \\
& \mathbf{P}_{\mathbf{0}}=\left(\begin{array}{ll}
0 & 0 \\
1 & 0
\end{array}\right) \text { if } n_{x, y}(\mathrm{R})<0\left(\text { or } n_{1}<n_{2}\right)
\end{aligned}
$$

Because $k=2$ we obtain:

$$
\begin{aligned}
& \text { if } n_{1}>n_{2}, \text { then } \mathrm{K}\left(R_{i} \oplus R_{0}\right)= \begin{cases}1 & \text { for } i=1, \ldots, n_{1} \\
0 & \text { otherwise }\end{cases} \\
& \text { if } n_{2}>n_{1} \text {, then } \mathrm{K}\left(R_{i} \oplus R_{0}\right)= \begin{cases}1 & \text { for } i=n_{1}+1, \ldots, n_{1}+n_{2} \\
0 & \text { otherwise }\end{cases} \\
& \text { if } n_{1}=n_{2} \text {, then } \mathrm{K}\left(R_{i} \oplus R_{0}\right)= \begin{cases}1 & \text { for } i=n_{1}+n_{2}+1, \ldots, N \\
0 & \text { otherwise }\end{cases}
\end{aligned}
$$

Therefore

$$
\partial_{\mathrm{K}}\left(\mathrm{R}, R_{0}\right)= \begin{cases}\frac{n_{1}}{N} & \text { if } n_{1}>n_{2}, \\ \frac{n_{2}}{N} & \text { if } n_{2}>n_{1}, \\ \frac{N-n_{1}-n_{2}}{N}=1-2 \frac{n_{1}}{N} & \text { if } n_{1}=n_{2} .\end{cases}
$$

This means that under either RCM-B or RCM-C, total lack of consensus only happens under a precise fifty-fifty division among all the voters (half prefer $x$ strictly over $y$, half the other way around), which is commonly agreed upon (see e.g., Alcalde and Vorsatz, 2008, pp. 2-3). Obviously when $k=2$ and $n_{1}+n_{2}=N$ (i.e., the dichotomous and binary case) one has 


$$
\partial_{\mathrm{K}}\left(\mathrm{R}, R_{0}\right)= \begin{cases}\frac{n_{1}}{N} & \text { if } n_{1}>n_{2} \\ \frac{n_{2}}{N} & \text { if } n_{2}>n_{1} \\ 0 & \text { if } n_{1}=n_{2}\end{cases}
$$

and an odd number of voters can not produce zero consensus under these models.

\section{Normal referenced consensus measures: a critical analysis of their properties}

In this Section, $\mathbf{M}=\left(\mathrm{C}, \partial_{\mathrm{M}}^{p}\right)$ denotes a normal referenced consensus measure with a $\mu^{p}(\mathrm{M})$ RMF. In other words, $\partial_{\mathrm{M}}^{p}$ is based on a conventional consensus measure $\mathrm{M}$ and is computed as a $p$-generalized mean according to (1). We proceed to check that such a model agrees with certain axioms that are in common use in the literature. At this point we remark that Axioms 1 to 3 below hold true in the larger class of referenced consensus measures. Finally, a critical analysis of other ad-hoc properties is performed.

\subsection{Some properties of referenced consensus measures}

The following axiom is trivial from the definition of a referenced consensus measure. It means that maximum consensus is reached under commonly held preferences across agents.

Axiom $1 \mathbf{M}$ is unanimous if for each constant profile $R$ it is true that $\nabla_{\mathbf{M}}(R)=1$.

Similarly, Proposition 1 below proves that the following property obtains:

Axiom $2 \mathbf{M}$ is anonymous if for each permutation of the voters $\sigma$ and each profile $\mathrm{R}$, it is true that $\nabla_{\mathbf{M}}(\mathrm{R})=\nabla_{\mathbf{M}}\left(\mathrm{R}^{\sigma}\right)$.

As is apparent, anonymity of a normal referenced consensus measure means that the consensus measure does not change if we rename the voters.

Proposition 1 Any $\mathbf{M}$ is anonymous.

Proof. This holds because $\mathbf{M}$ has properties 1.b) and 2.b). Specifically, 


$$
\nabla_{\mathbf{M}}(\mathrm{R})=\partial(\mathrm{R}, \mathrm{C}(\mathrm{R})) \stackrel{2 . b)}{=} \partial\left(\mathrm{R}^{\sigma}, \mathrm{C}(\mathrm{R})\right) \stackrel{1 . b)}{=} \partial\left(\mathrm{R}^{\sigma}, \mathrm{C}\left(\mathrm{R}^{\sigma}\right)\right)=\nabla_{\mathbf{M}}\left(\mathrm{R}^{\sigma}\right)
$$

In particular, both RCM-B and RCM-C satisfy Axiom 2. We now argue that normal referenced consensus measures verify the following property too:

Axiom $3 \mathbf{M}$ is neutral if the consensus measure does not change when we rename the candidates.

Proposition 2 Any $\mathbf{M}$ is neutral.

Proof. From properties 1.c) and 2.c) we obtain

$$
\nabla_{\mathbf{M}}(\mathrm{R})=\partial(\mathrm{R}, \mathrm{C}(\mathrm{R})) \stackrel{2 . c)}{=} \partial\left({ }^{\pi} \mathrm{R},{ }^{\pi} \mathrm{C}(\mathrm{R})\right) \stackrel{1 . c)}{=} \partial\left({ }^{\pi} \mathrm{R}, \mathrm{C}\left({ }^{\pi} \mathrm{R}\right)\right)=\nabla_{\mathbf{M}}\left({ }^{\pi} \mathrm{R}\right) .
$$

In order to introduce a further property of normal referenced consensus measures, we first give some notation. For each profile $\mathrm{R}=\left(R_{1}, \ldots, R_{N}\right)$ and $m \in \mathrm{N}$ we denote

$$
{ }^{m} \mathrm{R}=\left(R_{1}, \ldots, R_{1}, R_{2}, \ldots, R_{2}, \ldots, R_{N}, \ldots, R_{N}\right)
$$

that we call an $m$-replication of the profile $\mathrm{R}$. Then we say that the consensus function $\mathrm{C}$ verifies replication if $C(R)=C\left({ }^{m} R\right)$ throughout. This means that for each fixed society, the same consensus ordering is proposed if we repeatedly clone it. Likewise we define:

Axiom $4 \mathrm{M}$ verifies the replication axiom if for each profile $R$ and $m \in N$ it is true that $\nabla_{\mathbf{M}}(\mathrm{R})=\nabla_{\mathbf{M}}\left({ }^{m} \mathrm{R}\right)$.

Coupled with Axiom 2, this property is the analogue of the replication axiom in Alcalde and Vorsatz (2008) ${ }^{3}$. They interpret it as an invariance property asking that exact replications of a society are attached the same level of coherence as the original. The following result checks the model under inspection against Axiom 4:

Proposition 3 Given $\mathbf{M}=\left(\mathrm{C}, \partial_{\mathrm{M}}^{p}\right)$, if $\mathbf{C}$ verifies replication then $\mathbf{M}$ satisfies the replication axiom.

\footnotetext{
${ }^{3}$ These authors acknowledge inspiration by the scale invariance axiom in Allison (1978) characterization of the Gini index.
} 
Proof. Let us fix a profile $\mathrm{R}=\left(R_{1}, \ldots, R_{N}\right)$ and $m \in \mathrm{N}$. By definition of ${ }^{m} \mathrm{R}$, it is clear that for any complete peorder $R$ one has

$$
\begin{aligned}
\partial_{\mathrm{M}}^{p}\left({ }^{m} \mathrm{R}, R\right) & =\left(\frac{\sum_{i=1}^{N} \mathrm{M}\left(R_{i} \oplus R\right)^{p}+\ldots \ldots . .+\sum_{i=1}^{N} \mathrm{M}\left(R_{i} \oplus R\right)^{p}}{m \cdot N}\right)^{1 / p} \\
& =\left(\frac{\sum_{i=1}^{N} \mathrm{M}\left(R_{i} \oplus R\right)^{p}}{N}\right)^{1 / p}=\partial_{\mathrm{M}}^{p}(\mathrm{R}, R) .
\end{aligned}
$$

Thus because $\mathrm{C}$ satisfies replication we finally obtain

$$
\nabla_{\mathbf{M}}\left({ }^{m} \mathrm{R}\right)=\partial_{\mathrm{M}}^{p}\left(\mathrm{R}, \mathrm{C}\left({ }^{m} \mathrm{R}\right)\right)=\partial_{\mathrm{M}}^{p}(\mathrm{R}, \mathrm{C}(\mathrm{R}))=\nabla_{\mathbf{M}}(\mathrm{R}) .
$$

Corollary 1 Both RCM-B and RCM-C verify the replication axiom.

Proof. By Proposition 3 it suffices to prove that both Borda and Copeland rankings satisfy replication. Let us fix a profile $\mathrm{R}=\left(R_{1}, \ldots, R_{N}\right)$ and $m \in \mathrm{N}$. We observe that because $\mathbf{A}\left({ }^{m} \mathrm{R}\right)=m \cdot \mathbf{A}(\mathrm{R})$ and $\overline{\mathbf{A}}\left({ }^{m} \mathrm{R}\right)=m \cdot \overline{\mathbf{A}}(\mathrm{R})$ the Borda ranking is preserved by $m$ replication of the profile. Further, the fact that $\widetilde{\mathbf{A}}(\mathrm{R})=\operatorname{sig}(\overline{\mathbf{A}}(\mathrm{R}))=\operatorname{sig}\left(\overline{\mathbf{A}}\left({ }^{m} \mathrm{R}\right)\right)=\widetilde{\mathbf{A}}\left({ }^{m} \mathrm{R}\right)$ implies that the Copeland ranking is preserved by $m$-replication of the profile.

The next Axiom captures the intuitively appealing property that the consensus measure should not change if all the agents simultaneously reverse their orderings of the alternatives:

Axiom $5 \mathrm{M}$ verifies reversal invariance if the reversal of any profile $R$, namely $R^{-1}$, produces the same consensus, i.e.,

$$
\nabla_{\mathbf{M}}(\mathrm{R})=\nabla_{\mathbf{M}}\left(\mathrm{R}^{-1}\right) \text { for each possible profile } \mathrm{R} \text {. }
$$

To discuss this property, we have to introduce some additional notations. A consensus function C satisfies the reversal property if $\mathrm{C}\left(R^{-1}\right)=\mathrm{C}(R)^{-1}$ for any complete peorder $R$. This means that when all voters in a profile reverse their rankings of the candidates then the outcome is reversed. A consensus measure $M$ verifies the reversal property if $M\left(R^{-1}\right)=M(R)$ for any 
profile of complete preorders. That is, the consensus measure is unchanged when the profile is reversed. Let us analyse this property in detail.

Proposition 4 Given $\mathbf{M}=\left(\mathrm{C}, \partial_{\mathrm{M}}^{p}\right)$, if $\mathrm{C}$ and $\mathrm{M}$ verify the reversal property, then $\mathbf{M}$ satisfies the reversal invariance axiom.

Proof. Since $\left(R_{i} \oplus \mathrm{C}(\mathrm{R})\right)^{-1}=R_{i}^{-1} \oplus \mathrm{C}(\mathrm{R})^{-1}$, by hypothesis we infer for all $i=1 \ldots N$ that $\mathrm{M}\left(R_{i} \oplus \mathrm{C}(\mathrm{R})\right)=\mathrm{M}\left(\left(R_{i} \oplus \mathrm{C}(\mathrm{R})\right)^{-1}\right)=\mathrm{M}\left(R_{i}^{-1} \oplus \mathrm{C}(\mathrm{R})^{-1}\right)=\mathrm{M}\left(R_{i}^{-1} \oplus \mathrm{C}\left(\mathrm{R}^{-1}\right)\right)$, and thus from definition of $\mu^{p}(\mathrm{M})$-RMF we conclude as follow

$$
\nabla_{\mathbf{M}}(\mathrm{R})=\partial_{\mathrm{M}}^{p}(\mathrm{R}, \mathrm{C}(\mathrm{R}))=\partial_{\mathrm{M}}^{p}\left(\mathrm{R}^{-1}, \mathrm{C}\left(\mathrm{R}^{-1}\right)\right)=\nabla_{\mathbf{M}}\left(\mathrm{R}^{-1}\right) .
$$

Our particular proposals in Section 3 verify this property too.

Corollary 2 Both RCM-B and RCM-C verify the reversal invariance axiom.

Proof. Because the Borda and the Copeland rules satisfy the reversal property (Saari and Merlin, 1996, Section 1) we only have to prove that Kemeny's measure verifies the reversal property. This is straightforward since $\mathrm{K}^{s, t}\left(R_{i} \oplus \mathrm{C}(\mathrm{R})\right)=\mathrm{K}^{s, t}\left(R_{i}^{-1} \oplus \mathrm{C}\left(\mathrm{R}^{-1}\right)\right)$ for each possible voter $i$ and candidates $S$ and $t$.

We now investigate if normal referenced consensus measures verify the following reinforcement property:

Axiom $6 \mathrm{M}$ verifies reinforcement if adding $\mathrm{C}(R)$ to the profile $R$ does not reduce the consensus, i.e.,

$$
\nabla_{\mathbf{M}}(\mathrm{R} \oplus \mathrm{C}(R)) \geq \nabla_{\mathbf{M}}(\mathrm{R}) \text { for each possible profile } \mathrm{R} \text {. }
$$

We proceed to state a criterion for satisfaction of this property that depends upon the behavior of $\mathrm{C}$, and then we check that RCM-B meets such criterion but RCM-C does not. We say that a consensus function $C$ verifies decision invariance if $C(R \oplus C(R))=C(R)$ for each profile $\mathrm{R}$. This means that the consensus ordering does not change if we add to the society a new agent whose preferences coincide with the previous consensus preorder. Under this restriction we obtain: 
Proposition 5 Given $\mathbf{M}=\left(\mathrm{C}, \partial_{\mathrm{M}}^{p}\right)$, if $\mathbf{C}$ verifies decision invariance then $\mathbf{M}$ verifies reinforcement.

Proof. Since $\mathrm{C}(\mathrm{R} \oplus \mathrm{C}(\mathrm{R}))=\mathrm{C}(\mathrm{R})$, and using $\mathrm{M}(\mathrm{C}(R) \oplus \mathrm{C}(R))=1$, one has

$$
\begin{aligned}
\nabla_{\mathbf{M}}(\mathrm{R} \oplus \mathrm{C}(\mathrm{R}))=\partial_{\mathrm{M}}^{p}(\mathrm{R} \oplus \mathrm{C}(\mathrm{R}), \mathrm{C}(\mathrm{R})) \\
=\left(\frac{\sum_{i=1}^{N} \mathrm{M}\left(R_{i}, \mathrm{C}(\mathrm{R})\right)^{p}+\mathrm{M}(\mathrm{C}(\mathrm{R}), \mathrm{C}(\mathrm{R}))^{p}}{N+1}\right)^{1 / p} \\
=\left(\frac{N}{N+1}\left[\partial_{\mathrm{M}}^{p}(\mathrm{R}, \mathrm{C}(\mathrm{R}))\right]^{p}+\frac{1}{N+1}\right)^{1 / p} \geq \partial_{\mathrm{M}}^{p}(\mathrm{R}, \mathrm{C}(\mathrm{R}))=\nabla_{\mathrm{M}}(\mathrm{R}),
\end{aligned}
$$

where the last inequality derives from the fact $\nabla_{\mathbf{M}}(R) \leq 1$. Such inequality becomes strict provided $\nabla_{\mathbf{M}}(\mathrm{R})<1$

In Proposition 6 below, an appeal to Proposition 5 permits us to prove that RCM-B verifies reinforcement. Remark 1 below shows that the Copeland rule does not verify decision invariance ${ }^{4}$.

Proposition 6 RCM-B verifies reinforcement.

Proof. We just need to prove that the Borda rule verifies the decision invariance property which in conjunction with Proposition 5, proves the assertion. Let us take the profile $\mathrm{R}$ and denote $R_{B}=\mathrm{C}_{\mathrm{B}}(\mathrm{R})$ with preference matrix $\mathbf{P}_{B}$. Recall that for $\overline{\mathbf{A}}(\mathrm{R})=\mathbf{A}(\mathrm{R})-(\mathrm{A}(\mathrm{R}))^{t}$, the sum of the cells in its $i$-th row is $\beta\left(x_{i}\right)$, the Borda score of alternative $x_{i}$. We claim $C_{B}\left(R \oplus C_{B}(R)\right)=C_{B}(R)$. These orders arise from the respective Borda scores, namely $\beta_{B}$ and $\beta$, obtained from $\overline{\mathbf{A}}\left(\mathrm{R} \oplus \mathrm{C}_{\mathrm{B}}(\mathrm{R})\right)$ and $\overline{\mathbf{A}}(\mathrm{R})$ by summing up the cells in their rows. Observe $\overline{\mathbf{A}}\left(\mathrm{R} \oplus \mathrm{C}_{\mathrm{B}}(\mathrm{R})\right)=\overline{\mathbf{A}}(\mathrm{R})+\mathbf{P}_{B}-\left(\mathbf{P}_{B}\right)^{t}$. By construction $\beta\left(x_{i}\right) \geq \beta\left(x_{j}\right)$ if and only if $X_{i} R_{B} X_{j}$. Because the sum of the cells in the $i$-th row of $\mathbf{P}_{B}-\left(\mathbf{P}_{B}\right)^{t}$ is $\#\left\{x_{l} \in X: x_{i} P_{B} x_{l}\right\}-\#\left\{x_{l} \in X: x_{l} P_{B} x_{i}\right\}$, one has that $x_{i} R_{B} x_{j}$ if and only if the sum of

\footnotetext{
${ }^{4}$ The authors are grateful to an anonymous referee for detecting an error in an earlier version of the paper
} 
the cells in the $i$-th row of $\mathbf{P}_{B}-\left(\mathbf{P}_{B}\right)^{t}$ is greater or equal than the sum of the cells in the $j$-th row of $\mathbf{P}_{B}-\left(\mathbf{P}_{B}\right)^{t}$. This proves our claim $\beta_{B}\left(x_{i}\right) \geq \beta_{B}\left(x_{j}\right)$ if and only if $\beta\left(x_{i}\right) \geq \beta\left(x_{j}\right)$ throughout.

Remark 1 The Copeland rule fails to verify the decision invariance property. Consider the profile $\mathrm{R}$ of Example 4, then the Copeland rule provides the preorder $R$ such that wIzPxIy. For the profile $\mathrm{R} \oplus R$, the preorder $R^{\prime}$ that is prescribed by the Copeland rule is $z P^{\prime} w P^{\prime} y P^{\prime} x$ because

$$
\mathbf{P}=\left(\begin{array}{llll}
0 & 0 & 0 & 0 \\
0 & 0 & 0 & 0 \\
1 & 1 & 0 & 0 \\
1 & 1 & 0 & 0
\end{array}\right) \text { is the preference matrix of } R(v ., \text { Example 5) }
$$

hence

$$
\mathbf{A}(\mathrm{R} \oplus R)=\left(\begin{array}{llll}
0 & 1 & 2 & 1 \\
2 & 0 & 1 & 1 \\
2 & 3 & 0 & 2 \\
3 & 3 & 1 & 0
\end{array}\right) \text { and } \widetilde{\mathbf{A}}(\mathrm{R} \oplus R)=\left(\begin{array}{rrrr}
0 & -1 & 0 & -1 \\
1 & 0 & -1 & -1 \\
0 & 1 & 0 & 1 \\
1 & 1 & -1 & 0
\end{array}\right)
$$

This means $C_{C}\left(R \oplus C_{C}(R)\right) \neq C_{C}(R)$.

In order to prove another interesting property of a suitable subclass of normal referenced consensus measures we need some previous elaboration. The consensus function $\mathrm{C}$ verifies responsiveness if for every $R^{\prime} \in W(X)$ and $\mathrm{R} \in W(X)^{N}$, the following equality holds eventually (i.e., for all sufficiently large $m$ ):

$$
\mathrm{C}\left(\mathrm{R} \oplus R^{\prime} \oplus \ldots{ }^{m} . . \oplus R^{\prime}\right)=R^{\prime}
$$

We proceed to prove that the Borda rule and the Copeland rule verify a restricted version of this property, namely restricted responsiveness: for every $R^{\prime} \in L(X)$ and $\mathrm{R} \in W(X)^{N}$, equation (2) holds eventually.

Lemma 1 The Borda and Copeland rules verify restricted responsiveness. 
Proof. We fix $X=\left\{x_{1}, \ldots, x_{k}\right\}, R^{\prime} \in L(X)$, and $\mathrm{R} \in W(X)^{N}$.

Firstly we analyse the Borda rule. Given $x_{s} \neq x_{t}$ we can assume $x_{s} P^{\prime} x_{t}$ without loss of generality. Now irrespective of the Borda score that $\mathrm{R}$ attaches to them -namely, $\beta_{\mathrm{R}}\left(x_{s}\right)$ and $\beta_{\mathrm{R}}\left(x_{t}\right)-$ it must be the case that for sufficiently large $m$ the Borda score with respect to $\mathrm{R}^{m}=\mathrm{R} \oplus R^{\prime} \oplus \ldots{ }^{m} . . \oplus R^{\prime}$-which we denote by $\beta_{\mathrm{R}^{m}}-$ is strictly higher for $x_{s}$, since

$$
\beta_{\mathrm{R}^{m}}\left(x_{s}\right)-\beta_{\mathrm{R}^{m}}\left(x_{t}\right) \geq m+\beta_{\mathrm{R}}\left(x_{s}\right)-\beta_{\mathrm{R}}\left(x_{t}\right)
$$

If $m_{0}$ is such that $m>m_{0}$ implies $m+\beta_{\mathrm{R}}\left(x_{s}\right)-\beta_{\mathrm{R}}\left(x_{t}\right)>0$ then $m>m_{0}$ implies that the ordering between $x_{s}$ and $x_{t}$ according to $\mathrm{R}^{m}=\mathrm{R} \oplus R^{\prime} \oplus \ldots{ }^{m} . . \oplus R^{\prime}$ coincides with its ordering according to $R^{\prime}$. Because there are finitely many pairs in $\mathbf{K}$, this conclusion can be simultaneously reached for every pair $X_{s} \neq X_{t}$ of elements in $X$.

We now analyse the Copeland rule. Given $x_{s} \neq x_{t}$ we can assume $x_{s} P^{\prime} x_{t}$ without loss of generality. It is clear that for sufficiently large $m$ the alternative $x_{s}$ beats $x_{t}$ by strict simple majority according to $\mathrm{R}^{m}=\mathrm{R} \oplus R^{\prime} \oplus \ldots{ }^{m} . . R^{\prime}$. Formally: denote by $\kappa^{\prime}$ the Copeland score of the profile with the linear order $R^{\prime}$ only, and by $\kappa_{m}$ the Copeland score of the profile $\mathrm{R}^{m}$, then $\kappa^{\prime}\left(x_{s}\right)>\kappa^{\prime}\left(x_{t}\right)$ and it is eventually true that $\kappa_{m}\left(x_{s}\right)=\kappa^{\prime}\left(x_{s}\right)>\kappa^{\prime}\left(x_{t}\right)=\kappa_{m}\left(x_{t}\right)$. Now the argument goes through as above.

Responsiveness can not be guaranteed in Lemma 1: even in the simplest non-trivial instance where there are two candidates both the Borda rule and the Copeland rule fail to be responsive as the next example shows.

Example 8 Suppose $X=\{x, y\}$ thus $k=2$. Let $\mathrm{R}=\left(R_{1}\right)$ be the profile of one linear order given by $x P_{1} y$. We also let $R^{\prime}$ be the complete preorder with $x I^{\prime} y$, which is not a linear order. Then $\mathrm{C}_{\mathrm{B}}\left(\mathrm{R} \oplus R^{\prime} \oplus \ldots{ }^{m} . . \oplus R^{\prime}\right)=\mathrm{C}_{\mathrm{C}}\left(\mathrm{R} \oplus R^{\prime} \oplus \ldots{ }^{m} . . \oplus R^{\prime}\right)=R_{1}$ for each $m$, that is, both the Borda and Copeland methods suggest the consensus ordering $R_{1} \neq R^{\prime}$. The reason is that irrespective of $m$, the Borda score of $x$ is a unit higher than the Borda score of $y$, and the Copeland score of $x$ is 1 but the Copeland score of $y$ is 0 . 
We are now ready to define:

Axiom $7 \mathbf{M}$ verifies convergence to unanimity if for every $R^{\prime} \in W(X)$,

$$
\lim _{m \rightarrow \infty} \nabla_{\mathbf{M}}\left(\mathrm{R} \oplus R^{\prime} \oplus \ldots{ }^{m} \oplus R^{\prime}\right)=1 .
$$

Axiom $8 \quad \mathbf{M}$ verifies restricted convergence to unanimity if for every $R^{\prime} \in L(X)$ and $\mathrm{R} \in W(X)^{N}, \lim _{m \rightarrow \infty} \nabla_{\mathbf{M}}\left(\mathrm{R} \oplus R^{\prime} \oplus \ldots{ }^{m} \oplus R^{\prime}\right)=1$

We proceed to elucidate to which extent normal referenced consensus measures verify convergence to unanimity, with an especial attention to the RCM-B and RCM-C cases.

Proposition 7 Given $\mathbf{M}=\left(\mathrm{C}, \partial_{\mathrm{M}}^{p}\right)$, if $\mathbf{C}$ is responsive (resp., restrictedly responsive) then M verifies Axiom 7 (resp., Axiom 8). In particular, RCM-B and RCM-C verify restricted convergence to unanimity.

Proof. Suppose $\mathrm{C}$ is responsive, that is, for every $R^{\prime} \in W(X)$ and $\mathrm{R} \in W(X)^{N}$ the equality $\mathrm{C}\left(\mathrm{R} \oplus R^{\prime} \oplus \ldots{ }^{m} . . \oplus R^{\prime}\right)=R^{\prime}$ is eventually true. Then one has

$$
\begin{aligned}
\lim _{m \rightarrow \infty} \nabla_{\mathbf{M}}\left(\mathrm{R} \oplus R^{\prime} \oplus \ldots \oplus R^{\prime}\right) & =\lim _{m \rightarrow \infty} \partial_{\mathrm{M}}^{p}\left(\mathrm{R} \oplus R^{\prime} \oplus \ldots \oplus R^{\prime}, \mathrm{C}\left(\mathrm{R} \oplus R^{\prime} \oplus \ldots \oplus R^{\prime}\right)\right) \\
& =\lim _{m \rightarrow \infty} \partial_{\mathrm{M}}^{p}\left(\mathrm{R} \oplus R^{\prime} \oplus \ldots \oplus R^{\prime}, R^{\prime}\right) \\
& =\lim _{m \rightarrow \infty}\left(\frac{\sum_{i=1}^{N} \mathrm{M}\left(R_{i} \oplus R^{\prime}\right)^{p}+m}{N+m}\right)^{1 / p} \\
& =\lim _{m \rightarrow \infty}\left(\frac{N}{N+m}\left[\partial_{\mathrm{M}}^{p}\left(\mathrm{R}, R^{\prime}\right)\right]^{p}+\frac{m}{N+m}\right)^{1 / p}=1
\end{aligned}
$$

where we are using that $\mathrm{M}\left(R^{\prime} \oplus R^{\prime}\right)=1$ and $\partial_{\mathrm{M}}^{p}\left(\mathrm{R}, R^{\prime}\right) \leq 1$.

The case of a restrictedly responsive consensus function is proved analogously. In particular, Lemma 1 ensures that RCM-B and RCM-C verify restricted convergence to unanimity.

We now investigate the relationship between the consensus of two parts of the society and the 
consensus of the entire society. It is easy to show that our general model does not verify the following very demanding property: when the population is divided into two (or more) groups with the same consensus, the overall consensus is also the same. In order to better link the consensus of different groups we just need that they select the same collective preference. In this case we can prove that the overall consensus should be a magnitude in between the minimun and the maximun consensus of the parts, a property called compensativeness (see Grabisch et al., 2009):

Axiom $9 \mathbf{M}$ verifies compensativeness if for every pair of profiles $\mathrm{R}$ and $\mathrm{R}^{\prime}$ with $C(R)=C\left(R^{\prime}\right)$ it is true that

$$
\min \left\{\nabla_{\mathbf{M}}(\mathrm{R}), \nabla_{\mathbf{M}}\left(\mathrm{R}^{\prime}\right)\right\} \leq \nabla_{\mathbf{M}}\left(\mathrm{R} \oplus \mathrm{R}^{\prime}\right) \leq \max \left\{\nabla_{\mathbf{M}}(\mathrm{R}), \nabla_{\mathbf{M}}\left(\mathrm{R}^{\prime}\right)\right\} .
$$

To analyse this property it is convenient to introduce the following concept. A consensus function $C$ is consistent if: $R, R^{\prime}$ are such that $C(R)=C\left(R^{\prime}\right)$ implies $C\left(R \oplus R^{\prime}\right)=C(R)=C\left(R^{\prime}\right)$. Proposition 8 below checks our model against Axiom 9.

Proposition 8 If $C$ is consistent then $\mathbf{M}=\left(C, \partial_{M}^{p}\right)$ verifies Axiom 9.

Proof. Let $\mathrm{R} \in W(X)^{N}$ and $\mathrm{R}^{\prime} \in W(X)^{N^{\prime}}$ be two profiles satisfying $\mathrm{C}(\mathrm{R})=\mathrm{C}\left(\mathrm{R}^{\prime}\right)$. Since $\mathrm{C}$ is consistent, we infer

$$
\begin{aligned}
\nabla_{\mathbf{M}}\left(\mathrm{R} \oplus \mathrm{R}^{\prime}\right)^{p} & =\frac{1}{N+N^{\prime}} \sum_{i=1}^{N+N^{\prime}} \mathrm{M}\left(\left(\mathrm{R} \oplus \mathrm{R}^{\prime}\right)_{i}, \mathrm{C}\left(\mathrm{R} \oplus \mathrm{R}^{\prime}\right)\right)^{p} \\
& =\frac{1}{N+N^{\prime}}\left\{\sum_{i=1}^{N} \mathrm{M}\left(\mathrm{R}_{i}, \mathrm{C}(\mathrm{R})\right)^{p}+\sum_{i=N+1}^{N+N^{\prime}} \mathrm{M}\left(\mathrm{R}_{i}^{\prime}, \mathrm{C}\left(\mathrm{R}^{\prime}\right)\right)^{p}\right\} \\
& =\frac{N}{N+N^{\prime}} \nabla_{\mathbf{M}}(\mathrm{R})^{p}+\frac{N^{\prime}}{N+N^{\prime}} \nabla_{\mathbf{M}}\left(\mathrm{R}^{\prime}\right)^{p} .
\end{aligned}
$$

Using this equality, it is straightforward to show

$$
\min \left\{\nabla_{\mathbf{M}}(\mathrm{R})^{p}, \nabla_{\mathbf{M}}\left(\mathrm{R}^{\prime}\right)^{p}\right\} \leq \nabla_{\mathbf{M}}\left(\mathrm{R} \oplus \mathrm{R}^{\prime}\right)^{p} \leq \max \left\{\nabla_{\mathbf{M}}(\mathrm{R})^{p}, \nabla_{\mathbf{M}}\left(\mathrm{R}^{\prime}\right)^{p}\right\} .
$$

Finally, we conclude by monotonicity of the function $f(x)=x^{1 / p}$.

Now Proposition 9 below proves that the RCM-B proposal verifies compensativeness, and Remark 2 below proves that the Copeland rule is not consistent.

Proposition 9 RCM-B verifies compensativeness. 
Proof. We just need to prove that the Borda rule is consistent, which in conjunction with Proposition 8, proves the assertion. Let $\mathrm{R} \in W(X)^{N}$ and $\mathrm{R}^{\prime} \in W(X)^{N^{\prime}}$ be two profiles with $C_{B}(R)=C_{B}\left(R^{\prime}\right)$. We observe that because $\bar{A}\left(R \oplus R^{\prime}\right)=\overline{\mathbf{A}}(R)+\bar{A}\left(R^{\prime}\right)$ the Borda scores satisfy

$$
\beta_{\mathrm{R} \oplus \mathrm{R}^{\prime}}\left(x_{i}\right)=\beta_{\mathrm{R}}\left(x_{i}\right)+\beta_{\mathrm{R}^{\prime}}\left(x_{i}\right), \quad \text { for all } x_{i} \in X .
$$

Since $C_{B}(R)=C_{B}\left(R^{\prime}\right)$ the scores $\beta_{R}$ and $\beta_{R^{\prime}}$ rank the alternatives in the same way. Observe that the sum of these scores $\beta_{\mathrm{R} \oplus \mathrm{R}^{\prime}}=\beta_{\mathrm{R}}+\beta_{\mathrm{R}^{\prime}}$ also produce the same ranking. Therefore we deduce $C_{B}(R)=C_{B}\left(R^{\prime}\right)=C_{B}\left(R \oplus R^{\prime}\right)$.

Remark 2 The Copeland rule fails to satisfy the consistency property. Suppose $X=\left\{x_{1}, x_{2}, x_{3}\right\}$ thus $k=3$. Let $\mathrm{R}=\left(R_{1}, R_{2}, R_{3}, R_{4}, R_{5}\right)$ and $\mathrm{R}^{\prime}=\left(R_{6}, R_{7}, R_{8}\right)$ be profiles of preorder given by:

$$
\begin{array}{llll}
y I_{1} z P_{1} x & z P_{2} y P_{2} x & y P_{3} x P_{3} z & x P_{4} z P_{4} y \\
x P_{5} z P_{5} y & x P_{6} y P_{6} z & y P_{7} z P_{7} x & z P_{8} x P_{8} y
\end{array}
$$

Some simple computations yield that both $C_{C}(R)$ and $C_{C}\left(R^{\prime}\right)$ are the indiference preorder $x I y I z$. Under consistency the consensus function $\mathrm{C}_{\mathrm{C}}$ on profile $\mathrm{R} \oplus \mathrm{R}^{\prime}$ would associate the same indifference preorder. However $C_{C}\left(R \oplus R^{\prime}\right)$ is the linear order for wich $z P x P y$.

Remark 3 If a consensus function $C$ satisfies the consistency property then $C$ verifies replication and decision invariance.

The following Axiom 10 is a weaker version of Axiom 9. It claims that if the society is composed by groups with the same collective opinion and consensus, then the overall consensus does not change.

Axiom $10 \mathrm{M}$ verifies quasi-consistency if for every pair of profiles $R$ and $R^{\prime}$ with $\mathrm{C}(\mathrm{R})=\mathrm{C}\left(\mathrm{R}^{\prime}\right)$ and $\nabla_{\mathbf{M}}(\mathrm{R})=\nabla_{\mathbf{M}}\left(\mathrm{R}^{\prime}\right)$ it is true that

$$
\nabla_{\mathbf{M}}(\mathrm{R})=\nabla_{\mathbf{M}}\left(\mathrm{R}^{\prime}\right)=\nabla_{\mathbf{M}}\left(\mathrm{R} \oplus \mathrm{R}^{\prime}\right) .
$$


It is clear that RCM-B verifies quasi-consistency, because in particular Proposition 8 assures that if $\mathbf{C}$ is consistent then $\mathbf{M}=\left(\mathbf{C}, \partial_{\mathrm{M}}^{p}\right)$ verifies Axiom 10 .

\subsection{On other requirements of referenced consensus measures}

The literature on measurement of consensus has dealt with other desirable properties that we briefly analyse in this Subsection. Axiom 11 below requests that null and full consensus are possible.

Axiom $11 M$ verifies full range if there are two profiles $R$ and $R^{\prime}$ such that $\nabla_{M}(R)=0$, $\nabla_{M}\left(\mathrm{R}^{\prime}\right)=1$

Neither RCM-B nor RCM-C verify this property in the sense that zero consensus is impossible for particular values of $N$ as seen in Subsection 3.3.

Similarly, we proceed to analyze the property of Monotonicity, whose formal definition is given in Alcalde and Vorsatz (2008). Intuitively it say as follows. Suppose that you measure the consensus in a society. Now one agent reverses her/his opinion about the ordering of one particular pair of alternatives only ${ }^{5}$. If the alternative that the agent favours after the change beats the other alternative in a pairwise comparison for the rest of the society then the consensus should increase. And if both alternatives tie in a pairwise comparison for the rest of the society then the consensus should not vary after the change.

Examples 9 and 10 below show that RCM-B does not verify any of the two statements that jointly define Monotonicity. The same goes for Examples 11 and 12 regarding RCM-C.

Example 9 Suppose $X=\{x, y, z\}$ thus $k=3$. Let $\mathrm{R}=\left(R_{1}, R_{2}, R_{3}\right)$ be the profile of linear orders given by: $y P_{1} x P_{1} z, x P_{2} y P_{2} z, y P_{3} z P_{3} x$. Then $\mathrm{C}_{\mathrm{B}}(\mathrm{R})$ is $P_{1}$, that is, the Borda method produces $P_{1}$. Some simple computations yield $\nabla_{\mathbf{M}_{B}}(\mathrm{R})=\frac{7}{9}$.

Consider the profile $\mathrm{R}^{\prime}=\left(R_{1}^{\prime}, R_{2}, R_{3}\right)$ where $R_{1}^{\prime}$ is the linear order $y P_{1}^{\prime} z P_{1}^{\prime} x$. Under monotonicity this profile would have consensus $\frac{7}{9}$. However $\nabla_{\mathbf{M}_{B}}\left(\mathrm{R}^{\prime}\right)=\frac{5}{9}$ because $C_{B}\left(R^{\prime}\right)$

\footnotetext{
${ }^{5}$ Observe that this excludes from the analysis the case of a reversal of the order between $x$ and $y$ e.g., in $y I_{1} z P_{1} x$ or in $y P_{1} z P_{1} x$. These reversals modify the ordering between other pairs of alternatives too.
} 
is the complete preorder $R^{\prime}$ for which $y P^{\prime} x I^{\prime} z$.

Example 10 Suppose $X=\{x, y, z\}$ thus $k=3$. Let $\mathrm{R}=\left(R_{1}, R_{2}, R_{3}\right)$ be the profile of linear orders given by: $y P_{1} x P_{1} z, z P_{2} x P_{2} y, y P_{3} z P_{3} x$. Then $C_{B}(R)$ is $P_{3}$, that is, the Borda method produces $P_{3}$. Some simple computations yield $\nabla_{\mathbf{M}_{B}}(\mathrm{R})=\frac{2}{3}$.

Consider the profile $\mathrm{R}^{\prime}=\left(R_{1}^{\prime}, R_{2}, R_{3}\right)$ where $R_{1}^{\prime}$ is the linear order $y P_{1}^{\prime} z P_{1}^{\prime} x$. Under monotonicity this profile would yield a higher consensus. However $\nabla_{\mathbf{M}_{B}}\left(\mathrm{R}^{\prime}\right)=\frac{5}{9}$ because $\mathrm{C}_{\mathrm{B}}\left(\mathrm{R}^{\prime}\right)$ is the complete preorder $R^{\prime}$ for which $y I^{\prime} z P^{\prime} x$.

Example 11 Suppose $X=\{x, y, z\}$ thus $k=3$. Let $\mathrm{R}=\left(R_{1}, R_{2}, R_{3}\right)$ be the profile of linear orders given by: $x P_{1} y P_{1} z, y P_{2} x P_{2} z, z P_{3} y P_{3} x$. Then $\mathrm{C}_{\mathrm{C}}(\mathrm{R})$ is $P_{2}$, that is, the Copeland method produces $P_{2}$. Some simple computations yield $\nabla_{\mathbf{M}_{C}}(\mathrm{R})=\frac{2}{3}$.

Consider the profile $\mathrm{R}^{\prime}=\left(R_{1}^{\prime}, R_{2}, R_{3}\right)$ where $R_{1}^{\prime}$ is the linear order $x P_{1}^{\prime} z P_{1}^{\prime} y$. Under monotonicity this profile would have consensus $\frac{2}{3}$. However $\nabla_{\mathbf{M}_{C}}\left(\mathrm{R}^{\prime}\right)=0$ because $\mathrm{C}_{\mathrm{C}}\left(\mathrm{R}^{\prime}\right)$ is the complete preorder $R^{\prime}$ for which $x I^{\prime} y I^{\prime} z$.

Example 12 Suppose $X=\{x, y, z\}$ thus $k=3$. Let $\mathrm{R}=\left(R_{1}, R_{2}, R_{3}, R_{4}\right)$ be the profile of linear orders given by: $x P_{1} y P_{1} z, x P_{2} y P_{2} z, z P_{3} x P_{3} y, z P_{4} x P_{4} y$. Then $C_{c}(R)$ is the linear order $x P z P y$ (that is, the Copeland method produces $P_{1}$ ). Some simple computations yield $\nabla_{\mathbf{M}_{C}}(\mathrm{R})=\frac{2}{3}$.

Consider the profile $\mathrm{R}^{\prime}=\left(R_{1}^{\prime}, R_{2}, R_{3}, R_{4}\right)$ where $R_{1}^{\prime}$ is the linear order whose asymmetric part is $P$ above (that is, $x P_{1}^{\prime} z P_{1}^{\prime} y$ ). Under monotonicity this profile would yield a higher consensus. However $\nabla_{\mathbf{M}_{C}}\left(\mathrm{R}^{\prime}\right)=\frac{7}{12}$ because $\mathrm{C}_{\mathrm{C}}\left(\mathrm{R}^{\prime}\right)$ is the complete preorder $R^{\prime}$ for which $x I^{\prime} z P^{\prime} y$. 


\section{Related literature and concluding remarks}

We conclude our paper with the following comments on our approach to this controversial topic and related literature ${ }^{6}$.

1. We have explored the normative properties of a general class of measures of the cohesiveness in a society. The standard tendency in this regard reflects the intention to produce an absolute coefficient of consensus, and to supply their axiomatic characterizations. Neither of those possible indices are banned by our general concept of a referenced consensus measure. The normality property permitted us to restrict ourselves to a wide framework where many desirable properties of a measure of cohesiveness ensue. We have made an extensive discussion of properties with a particular attention to the analysis of societies that are divided into disjoint parts that produce the same collective decision; here and elsewhere we concluded that the Borda benchmark permits a much better aggregative behavior than Copeland. Besides, our model reconciles the measurement of magnitudes of (dis)agreement of preferences with social choice theory in the vein of earlier works like: Kemeny (1959), who proposes a social welfare ordering that maximizes the probability of agreement with a randomly selected member of the group; Baigent (1987), who shows that social welfare functions that verify certain proximity preservation property cannot both respect unanimity and be anonymous (see also Baigent, 1989; Nitzan, 1989; Klamler, 2005), who started comparing the Copeland rule to other aggregation procedures explicitly based on distance information; Meskanen and Nurmi (2006), who study how classical social welfare functions relate to distance functions between rankings and profiles; or Kemeny's generalization by Baldiga and Green (2011) and to a lesser extent, Klamler (2008) more general approach by choice functions, among others.

2. Alcalde and Vorsatz (2010) criticize the abuse of the intuitive approach by mean aggregators of pairwise individual comparisons of similarity. The bulk of their argument is that it is restrictive because it casts aside important information that only the whole preference profile captures, as Example 1 in Alcalde and Vorsatz (2010) appears to show. Our approach does not disregard that intuitive approach but we believe that it partially circumvents such supposed handicap for two reasons. For one thing, because it contrasts personal preferences with a social target and this can collect the global information of the profile. Let us consider their example, where $X=\{x, y, z\}$ are the alternatives, and a three-person society has the following profile $P$ of linear

\footnotetext{
${ }^{6}$ Martínez-Panero (2008) gives a concise survey of the different meanings that the term consensus has traditionally had, both for
} philosophical and formal disciplines. 
orders: $x P_{1} y P_{1} z, x P_{2} y P_{2} z, y P_{3} z P_{3} x$. They argue that cohesiveness should be higher at the following profile $\bar{P}$ than at $P: x \bar{P}_{1} y \bar{P}_{1} z, x \bar{P}_{2} z \bar{P}_{2} y, y \bar{P}_{3} x \bar{P}_{3} z$. Indeed some simple computations yield $\nabla_{\mathbf{M}_{B}}(\bar{P})=0.77>0.55=\nabla_{\mathbf{M}_{B}}(P)$. For another, they claim that a weakly higher weight has to be given to objects that are more important at the social level. Because Definition 3 relies on generic consensus measures it can be designed so as to prioritize the socially relevant alternatives as well as to gather global information of the profile. This indicates that some route of escape that does not reject the intuitive original approach can be found.

3. Our informational input is a profile of complete preorders, a more general framework than the usual linearity requirement where ties are not allowed. Specific welfare rules have been studied. Other possibilities come to mind immediately. For example, the appeal to choice functions as in Klamler (2008), or in other terms the use of other focal aggregation rules to obtain the social objective. Links can be drawn between these possibilities since for example, easy correspondences can be made between approval voting and its generalizations (Brams and Fishburn, 1992; Laslier and Sanver, 2010) and choice functions.

4. We do not advocate for the universal adequacy of our model. However the question that a measure of the cohesiveness of personal preferences is a convenient tool for the analyst has been exploited in Alcantud, de Andrés and Cascón (2011). There we compare the performance of the Borda and Copeland voting rules for small numbers of alternatives. A computational analysis reveals that an ex-ante advice on the rule that should be adopted can be made in the wake of the chances that one rule produces higher consensus than the other.

\section{Acknowledgements}

The authors thank the Editor of Journal of Microeconomics, Roberto Veneziani, and a referee of this journal for their valuable comments and suggestions. J. C. R. Alcantud, R. de Andrés Calle and J. M. Cascón acknowledge financial support by the Spanish Ministerio de Economía y Competitividad (Projects ECO2009-07682 and ECO2012-31933; Project ECO2012-32178; and Project CGL2011-29396-C03-02, respectively). J. M. Cascón acknowledges financial support by Junta de Castilla y León under Project SA266A12-2. 


\section{References}

1. Bosch, R. (2005). Characterizations of voting rules and consensus measures. Ph.D. thesis; Tilburg University.

2. Alcalde, J., Vorsatz, M. (2010). Do We Agree? Measuring the Cohesiveness of Preferences. Mimeo.

3. Kemeny, J. (1959). Mathematics without Numbers. Daedalus, 88: 577-591.

4. Kendall, M. (1962). Rank correlation methods. Hafner Publishing Co., NY.

5. Cook, W., Seiford, L. (1978). Priority Ranking and Consensus Formation. Manage. Sci., 24: 1721-1732.

6. Cook, W., Seiford, L. (1982). On the Borda-Kendall Consensus Method for Priority Ranking Problems. Manage. Sci., 28: 621-637.

7. Klamler, C. (2008). A Distance Measure for Choice Functions. Soc. Choice Welfare, 30: 419-425.

8. Baldiga, K.A., Green, J.R. (2011). Assent-Maximizing Social Choice. Soc. Choice Welfare, doi: 10.1007/s00355-011-0614-6.

9. Alcantud, J.C.R., de Andrés Calle, R., Cascón, J.M. (2011). A Unifying Model to Measure Consensus Solutions in a Society. Math. Comput. Model., doi:10.1016/j.mcm.2011.12.020.

10. McMorris, F.R., Powers, R.C. (2009). Consensus Rules Based on Decisive Families: The Case of Hierarchies. Math. Soc. Sci., 57: 333-338.

11. Suzumura, K. (1983). Rational choice, collective decisions and social welfare. Cambridge University Press, Cambridge.

12. Saari, D., Merlin, V.R. (1996). The Copeland Method I: Relationships and the Dictionary. Econ. Theory, 8: 51-76.

13. Alcalde, J., Vorsatz, M. (2008). On the Measurement of Consensus. Mimeo.

14. Allison, P. (1978) Measures of Inequality. Am. Sociol. Rev., 43: 865-880.

15. Grabisch, M., Marichal, J.L., Mesiar, R., Pap, E. (2009). Aggregation functions. Cambridge University Press, Cambridge.

16. Martínez-Panero, M. (2008). Approaches to consensus: The state of the art. In: Proceedings of the XIV Spanish Conference on Fuzzy Technologies and Logic (ESTYLF08) pp. 469-476. European Centre for Soft Computing.

17. Baigent, N. (1987). Preference Proximity and Anonymous Social Choice. Q. J. Econ., 102: $131-170$

18. Baigent, N. (1989). Some Further Remarks on Preference Proximity. Q. J. Econ., 104: $191-193$

19. Nitzan, S. (1989). More on the Preservation of Preference Proximity and Anonymous Social Choice. Q. J. Econ., 104: 187-190. 
20. Klamler, C. (2005). The Copeland Rule and Condorcet's Principle. Econ. Theory, 25: $745-749$.

21. Meskanen, T., Nurmi, H. (2006). Distance from consensus: A theme and variations. In: Mathematics and Democracy pp. 117-132. Springer.

22. Brams, S., Fishburn, P.C. (1992). Approval voting. Birkhauser, Boston.

23. Laslier, J.F., Sanver M.R. (Eds.) (2010). Handbook on approval voting. Springer. 